\title{
A Comparative Study of Local Net Modeling Using Machine Learning
}

\author{
Jackson Melchert, Boyu Zhang, and Azadeh Davoodi \\ University of Wisconsin - Madison \\ \{jmelchert,bzhang93, adavoodi\}@wisc.edu
}

\begin{abstract}
Local nets are by default ignored during global routing but can contribute to a high percentage (up to $30 \%$ ) of total number of nets in the design. Prior work proposed simple models for how local nets are routed and showed benefits such as better congestion analysis post-placement, integration with global routing, and better track assignment. In this work we study local net modeling using machine learning. Our model predicts utilization by local routes inside each global cell. We model this as a regression problem and as reference use local route utilization data from the detailed routing stage using a commercial tool. To solve the problem we identify suitable machine learning algorithms. Within our modeling, we study and rank different features which utilize various layout attributes. We identify the most beneficial features and show our model performs superior to prior work which were based on pin density and Steiner tree models. Our model also performs better for the subset of local nets which are routed in more than one global cell.
\end{abstract}

\section{CCS CONCEPTS}

- Computing methodologies $\rightarrow$ Machine learning; • Hardware $\rightarrow$ Very Large Scale Integration Design; Modeling and parameter extraction; Physical design (EDA);

\section{KEYWORDS}

Machine Learning, Local Nets, Global Routing

\section{ACM Reference Format:}

Jackson Melchert, Boyu Zhang, and Azadeh Davoodi. 2018. A Comparative Study of Local Net Modeling Using Machine Learning . In GLSVLSI '18: 2018 Great Lakes Symposium on VLSI, May 23-25, 2018, Chicago, IL, USA. ACM, New York, NY, USA, 6 pages. https://doi.org/10.1145/3194554.3194579

\section{INTRODUCTION}

Obtaining a routable layout and removing violations have turned into key physical design challenges in modern designs. Addressing these challenges has recently inspired new routability-driven placement algorithms, and better algorithms and metrics for global routing and violation-free track assignment [2, 3, 6, 9, 11-14].

Permission to make digital or hard copies of all or part of this work for personal or classroom use is granted without fee provided that copies are not made or distributed for profit or commercial advantage and that copies bear this notice and the full citation on the first page. Copyrights for components of this work owned by others than ACM must be honored. Abstracting with credit is permitted. To copy otherwise, or republish, to post on servers or to redistribute to lists, requires prior specific permission and/or a fee. Request permissions from permissions@acm.org.

GLSVLSI '18, May 23-25, 2018, Chicago, IL, USA

(C) 2018 Association for Computing Machinery.

ACM ISBN 978-1-4503-5724-1/18/05 ..\$ \$15.00

https://doi.org/10.1145/3194554.3194579
Local nets are one of the factors which challenge obtaining a routable and violation-free layout. They are by default ignored during global routing and track assignment. However, they contribute up to about $30 \%$ of the total number of nets in a design. Some recent works have considered modeling local nets using simple models and shown improvement in global routing, post-placement congestion analysis, and track assignment. However, the local net model itself has not been verified against how these nets actually get routed at the detailed routing stage.

In this work, we study modeling the local nets using machine learning. Our contributions are outlined below.

- Using local route utilization from the detailed routing stage as reference data, we use machine learning algorithms to create a model at the post-placement stage.

- We evaluate and rank various post-placement and postglobal-routing features to identify the ones which are most beneficial for modeling.

- We show that the use of post-placement features which utilize information about the neighboring global cells help improve the modeling accuracy.

- We show consistent improvement across all designs compared to prior work which used Steiner tree or pin density to predict the local nets. Our work is also the first to use local net data from the detailed routing stage for comparison.

In the remainder of the paper we first review preliminaries and prior work in Section 2. We discuss our modeling process in Section 3. This is followed by simulation results and conclusions.

\section{PRELIMINARIES AND PRIOR WORK}

\subsection{Preliminaries}

At the post-placement stage, we are given a 3-dimensional placement grid, $P=\left\{0, \ldots, X_{P}\right\} \times\left\{0, \ldots, Y_{P}\right\} \times\{0, \ldots, L\}$. The design consists of a set of nets $N=\left\{T_{1}, T_{2}, \ldots, T_{|N|}\right\}$, where each net $T_{n} \in$ $N$ consists of a set of pin locations on $P$. Before performing global routing, this detailed placement grid is replaced with a less-refined global routing grid, $G=\left\{0, \ldots, X_{G}\right\} \times\left\{0, \ldots, Y_{G}\right\} \times\{0, \ldots, L\}$.

The global routing grid is made of rectangular regions called global cells (gcells) where the coordinates of the placement grid fall inside or on the boundaries of the gcells. Each gcell contains a number of routing tracks which are uniformly spaced and the width of each track is known. Before performing global routing a grid-graph is created from the global routing where each gcell is represented by a vertex and the boundary of two adjacent gcells is represented by an edge known as global edge. Each global edge $e$ has a capacity $c_{e}$ representing the number of routing tracks that can pass the corresponding boundary of its two adjacent gcells. At this point the nets are divided into either local or global nets. A net $T_{\ell}$ is a local net if all its pins are located inside the same gcell. 
Otherwise if a net has its pins in two or more gcells then it is called a global net. Once global routing is performed, all global nets are routed and local nets are by default ignored. After global routing, each global edge will have a utilization $u_{e}$ representing the number of routing tracks used to route global nets in the corresponding boundary of its two adjacent gcells. The overflow of a global edge is defined as $o_{e}=\max \left(u_{e}-c_{e}, 0\right)$. An overflow higher than 0 indicates that the global edge is utilized beyond its capacity.

\subsection{Applications of Local Net Modeling}

Some recent works try to predict how local nets will be routed prior to performing global routing in order to have a more realistic estimate of the available capacity of each global edge. The work [12] models local routes and uses reduced global edge capacities (as some routing tracks are predicted to be utilized by local routes). This allows performing a more accurate global routing congestion analysis. Local-route-aware congestion analysis can also be performed prior to routing of global nets, during or after a routability-driven placement stage. This is assuming the global routing grid is known. The work [11] also models local routes and integrates their impact during global routing by introducing a vertex capacity in the global routing grid-graph. The capacity of a vertex is the area of its gcell after subtracting the utilization of its (expected) local routes.

In this work, we also aim to model local nets. Our goal is to model local route utilization for each gcell. Our model computes how much the routing tracks will be utilized after local nets are routed for each gcell. While local nets by definition have all their pins inside the same gcell, it is not necessary for a local net to be routed completely inside the same gcell and a small number of local nets may additionally be routed in the neighboring gcells. Our model accounts for this issue and generates a utilization number per gcell by local routes which are expected to fall inside it.

\subsection{Prior Work on Local Net Modeling}

Here we review two models of local route utilization which have been studied in prior work. First the authors in [12] model local route utilization inside each gcell to be proportional to the number of pins in the gcell. The proportionality factor is decided as a technology-dependent parameter. This model was developed based on the observation that each pin may be connected to a set of local wires, and the number of pins in a gcell is a good indicator of the number of local routes and a first-order estimate of the number of blocked routing tracks. The main benefit of this model is that it can be evaluated very quickly and at the post-placement stage because it only requires knowledge of pin locations and the global routing grid. This model is therefore useful for integration with a congestion analysis tool which may be evoked many times during routability-driven placement. In this work we made comparison with pin-density model in [12]. Since the technology parameter used in [12] did not match the 65nm node used by our designs, in our implementation of [12] we obtained the highest accuracy when we used linear regression to fit the pin density of each gcell to the local route utilization in the subset of designs which were used for training. This fitting was done using the same tool (Weka [5]) which implemented our machine learning algorithm. This approach produced the best results to implement [12] in our experimental setup. The proportionality factor was then used to validate during testing.

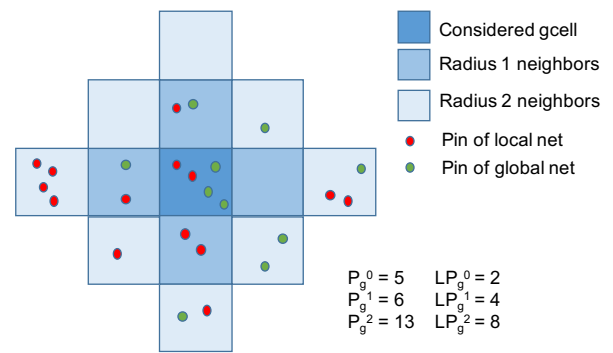

Figure 1: Example showing some post-placement features

The second model used for local net modeling is based on Steiner tree wirelength which has been used in [12]. In this model first a Steiner tree is generated based on the subset of the pins of a net which falls inside the same gcell. Therefore the net maybe a local net, or a global net in which some pins are located in the same gcell. Depending on where the edges of the Steiner tree fall, the corresponding routing tracks are computed and get blocked in the nearest global edge. These are done for the gcells in M2 and M3 based on the observation that the vast majority of local nets are routed on these two metal layers. After processing all the local nets, the number of blocked tracks at the boundary of each gcell is then computed and used to update the available capacity of each global edge. Similar to the pin density model, the work [12] used empirically-tuned parameters to scale the horizontal and vertical Steiner wirelength to the number of blocked tracks.

Compared to the pin density model, the Steiner wirelength model takes significantly longer to generate. Other variations of the Steiner wirelength model are also used in works such as $[9,10,13]$ and typically applied as a first step in congestion analysis and in track assignment. In this work, since we model local route utilization inside the gcell (and not number of blocked tracks), we directly use the Steiner wirelength of each local net which we generate using FLUTE [4]. The work [11] also models local nets by first routing them on metals M2 and M3 using a rip-up and reroute technique. The corresponding tracks are then blocked and used to update the available tracks inside each gcell.

\section{MACHINE LEARNING FRAMEWORK}

We first introduce the features collected for our modeling and rank them to identify the top ones. Next we discuss our modeling process.

\subsection{Feature Selection and Ranking}

For a considered gcell $g$ we define the following features.

- $P_{g}^{i}$ (numPins): cumulative pin density inside gcells at radius $i$ from $g$; a special case is $P_{g}^{0}$ which indicates pin density solely inside $g$. Also, $P_{g}^{1}$ corresponds to the immediate neighbors of $g, P_{g}^{2}$ corresponds to the neighbors of neighbors of $g$.

- $L P_{g}^{i}$ (numLocalNetPins): cumulative pin density of local nets inside gcells at radius $i$ from $g$; a special case is $L P_{g}^{0}$ which shows pin density of local nets inside $g$.

- $E W_{g}$ (estimatedWirelength): wirelength of local nets inside gcell $g$; to compute this feature, first the local pins inside $g$ are traversed, and a minimum bounding box is constructed between each pair of consecutive pins of the same local net. The sum of half-perimeter bounding box values across all local nets is computed as the estimated local wirelength of $g$. 


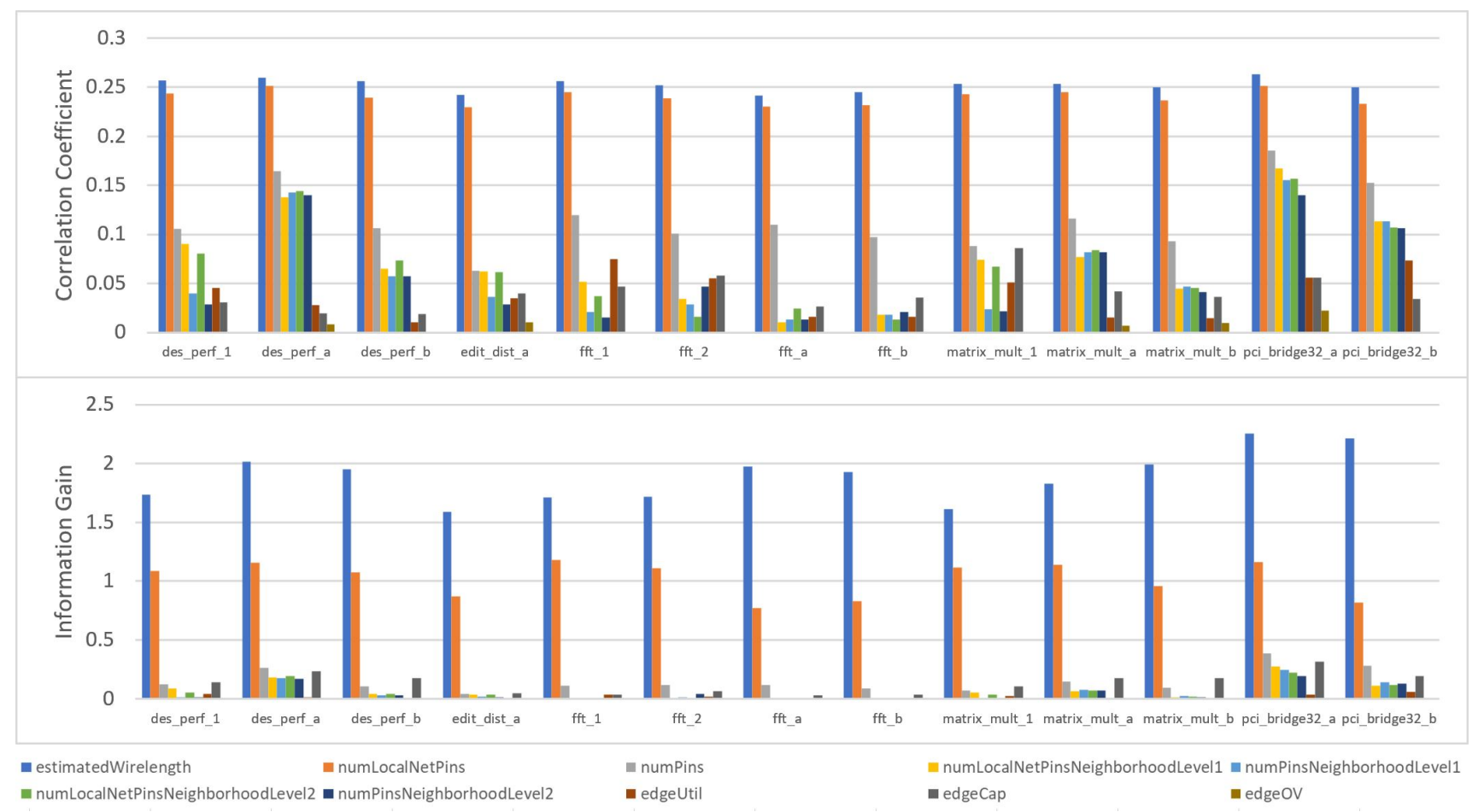

Figure 2: Comparison of layout features in predicting local route utilization using the IG and CC metrics in each benchmark

- $U_{g}$ (edgeUtil): sum of global routing edge utilizations corresponding to the four boundaries of $g$.

- $C_{g}$ (edgeCap): sum of global routing edge capacities corresponding to the four boundaries of $g$.

- $O_{g}$ (edgeOV): overflow of $g$ is computed based on its utilization and capacity: $\max \left(0, U_{g}-C_{g}\right)$.

In the above list, the features $P_{g}^{i}, L P_{g}^{i}, E W_{g}$ can be extracted at the post-placement stage, using information about the global routing grid (so the locations of the gcells are known). In our modeling framework, we collect the values of these features for $i=\{0,1,2\}$ so that a total of 7 post-placement features per g-cell. Figure 1 shows these features for a simple example and how various postplacement features (except $E W_{g}$ ) are computed. The 3 additional features $O_{g}, U_{g}$ and $C_{g}$ are collected at the post-global routing stage so they require to first perform global routing.

For the post-placement features, we did not include radius- 3 features because we observed that almost all local nets were routed within a radius of at most 1, i.e., they are routed either completely within the gcell or its immediate neighbors. So we just included radius 2 features in case of a potential domino effect when multiple gcells of high pin density are adjacent to each other.

Next, we used two metrics, information gain (IG) and correlation coefficient (CC), to measure the importance of each feature in predicting local route utilization. Information gain is the measure of reduction in the entropy of a variable achieved by learning the state of another random variable [8]. Correlation coefficient is a number that quantifies correlation between two variables. These metrics were measured with the Weka tool [5] using the data collected for each benchmark individually together with the reference data (of actual local route utilization) at the detailed routing stage.
In this work we used the 2015 ISPD contest benchmark suite [2]. Specifically, we used the subset of benchmarks implemented in $65 \mathrm{~nm}$ technology node all of which had 5 routing layers. We provide details about our experimental setup later.

Before measuring the IG and CC metrics we applied some preprocessing on the collected data. This was in order to highlight the difference in predictive ability of each feature. First, we discarded every gcell with 0 local route utilization to get a more meaningful representation of the predictive power of the features, especially in relation to the $\mathrm{CC}$ measurement.

The reason that removing these instances helped to differentiate features is that when a gcell has 0 local route utilization, it must have 0 local net pins, 0 estimated wirelength, and in most cases, values of 0 for every feature. This leads to very high correlation scores for every feature in benchmarks where there is little local route congestion, making it difficult to differentiate the features based on actual predictive power. Also, in these gcells, the prediction is trivial, and we observed that whenever the machine learning algorithm sees a gcell with 0 local net pins and 0 estimated wirelength, it will almost always predict 0 local route utilization.

Another preprocessing step that we took was to discretize the attributes into 10 equal frequency bins based on local route utilization. This again allowed to better compare the features in cases where local route utilization in all gcells was heavily skewed.

Figure 2 shows the ranking of each feature for each benchmark. For both IG and CC, the most important feature is estimatedWirelength $(E W)$, followed by numLocalNetPins $\left(L P^{0}\right)$. Both of these features are directly related to local route utilization, so it is not surprising that they contribute the most information in order to predict local route utilization. 


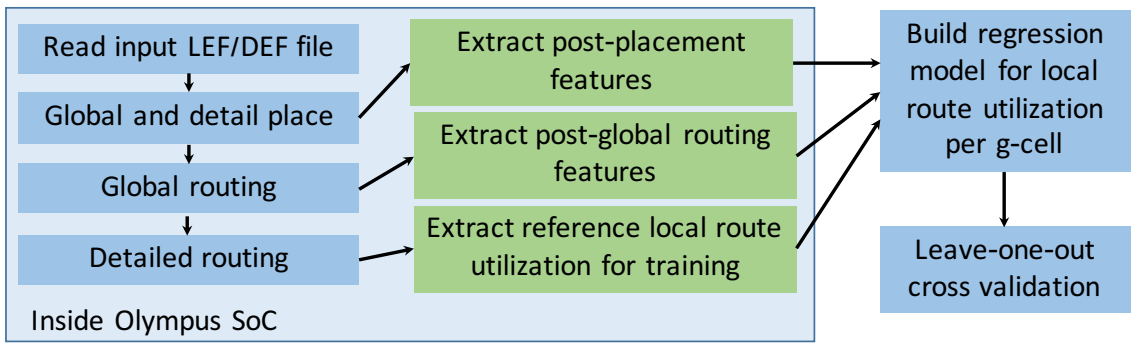

Figure 3: Flow chart of our modeling process

Post-global routing features clearly do not add much predictive ability to the model, and the fact that they need to be collected after completing global routing makes them even less useful. The level 1 and level 2 neighborhood features seem to be useful in some benchmarks but not in others, leading us to believe that in most cases, neighboring gcells do not impact local routes significantly.

We selected 5 features as input to our final machine learning algorithm: $E W_{g}, L P_{g}^{0}, P_{g}^{0}, L P_{g}^{1}, P_{g}^{1}$. While it is clear that $E W_{g}, L P_{g}^{0}$, and $P_{g}^{0}$ are the three most useful features overall, we wanted to include the radius 1 neighborhood features to help predict local route utilization in cases where local nets are routed outside of their own gcells, something that prior works did not consider.

\subsection{Our Modeling Process}

Figure 3 shows overview of our modeling process. We used Olympus SoC [1] to collect the values of these features in the $65 \mathrm{~nm}$ ISPD 2015 designs. First, the Olympus placement tool was used to do placement on each benchmark. The values of post-placement features were extracted at this point. Next, Olympus global router was called to generate a solution. The values of global routing features were extracted at this point. Finally, the Olympus track router was called to generate a detailed routing solution and the local route utilization as reference data. These features were all extracted using TCL scripting inside Olympus.

To extract the values of post-placement and post-global routing features, we first built a 2D-projection of the solution corresponding to that stage and computed the values of those features for the g-cells of that 2D-projected grid-graph. This allowed storing significantly smaller set of data.

To test each design, the values of the features were extracted from the remaining designs and used to train the model. This technique is known as leave-one-out cross validation. It not only enables us to have relatively more training instances to train our models, which is always good, but also gives us an unbiased evaluation about how well the algorithm works on current dataset. We note we used the same leave-one-out evaluation technique for implementing and comparing with other prior work in our experiments.

Our goal was to model local route utilization inside each gcell in the $2 \mathrm{D}$ projected global routing grid-graph. During our modeling, we experimented with different machine learning algorithms.

We note the reason we do feature extraction and modeling for the 2D-projected global routing graph is because we observed the vast majority of local routes were located on metal layers M2 and M3. Breakdown of the local route utilization between M2 and M3 followed a constant ratio across the benchmarks (about $48 \%$ on M2 and $40 \%$ on M3).

\section{EXPERIMENTAL RESULTS}

We used the modeling process explained in Figure 3 in Section 3. We experimented with the 65nm ISPD 2015 designs all of which had 5 routing layers. (Extension to evaluate designs from other technology nodes will be considered in our future work.) We used the tool Weka [5] which allowed data mining with various machine learning algorithms as well as simple linear regression (to implement prior work). We compared various machine learning algorithms and prior work. These were calculated in Olympus by comparing the predicted local route utilization versus the true local route utilization (at the detailed routing stage) per gcell.

For evaluation of accuracy we first define a Relative Absolute Error metric which is defined below.

$$
R A E_{\text {gcells }}=\frac{\sum_{\forall g \in \text { gcell }}\left|L U_{\text {approach }}^{g}-L U_{r e f}^{g}\right|}{\sum_{\forall g \in \text { gcell }}\left|E\left[L U_{\text {ref }}\right]-L U_{r e f}^{g}\right|}
$$

where $L U_{\text {approach }}^{g}$ is the local route utilization predicted by a specific approach for a gcell, $L U_{r e f}^{g}$ is the reference local utilization of the gcell and $E\left[L U_{r e f}\right]$ is the average of reference local utilizations across all the gcells. This error metric first computes an error in local utilization per gcell compared to its true value obtained from detailed routing. These errors are then added up across all gcells, and then the sum is normalized with respect to a reference value given in the denominator. The above formula for RAE allows handling cases when the true local route utilization of a gcell is 0 .

We first give a comparison of accuracy among various machine learning algorithms, then present comparison with prior works in terms of accuracy, runtime and outlier cases.

\subsection{Comparing Machine Learning Algorithms}

We trained and tested five machine learning models using the feature set we selected in Section 3.1 and the leave-one-out cross validation technique explained in Section 3.2. The machine learning algorithms are summarized below:

(1) RandomTree constructs a tree that considers K randomly chosen features at each node.

(2) MultilayerPerceptron creates a shallow neural network model. (3) DecisionTable constructs a simple decision tree with default majority mapping rule [7].

(4) RandomForest is an ensemble learning method for classification, regression and other tasks. During training, RandomForest constructs a multitude of decision trees where each decision tree is built based on a random sampling of the training instances and a random subset of the feature vector. 
Table 1: Relative Absolute Error over all gcells of different machine learning models trained with the top ranked features identified in Section 3.1. All results are obtained via leave-one-out cross validation.

\begin{tabular}{c|c|c|c|c|c}
\hline & RandomTree & MultilayerPerceptron & DecisionTable & RandomForest & REPTree \\
\hline mgc_des_perf_1 & $37.14 \%$ & $23.29 \%$ & $32.26 \%$ & $24.97 \%$ & $25.83 \%$ \\
mgc_des_perf_a & $10.48 \%$ & $40.82 \%$ & $16.16 \%$ & $7.39 \%$ & $7.40 \%$ \\
\hline mgc_des_perf_b & $21.38 \%$ & $14.31 \%$ & $27.88 \%$ & $14.62 \%$ & $14.24 \%$ \\
mgc_edit_dist_a & $16.15 \%$ & $25.99 \%$ & $27.04 \%$ & $12.90 \%$ & $12.89 \%$ \\
\hline mgc_fft_1 & $35.91 \%$ & $27.76 \%$ & $32.46 \%$ & $27.10 \%$ & $27.58 \%$ \\
mgc_fft_2 & $31.85 \%$ & $26.59 \%$ & $33.91 \%$ & $23.90 \%$ & $24.47 \%$ \\
mgc_fft_a & $9.64 \%$ & $28.08 \%$ & $28.86 \%$ & $7.39 \%$ & $7.63 \%$ \\
mgc_fft_b & $9.88 \%$ & $25.86 \%$ & $24.20 \%$ & $7.73 \%$ & $7.68 \%$ \\
mgc_matrix_mult_1 & $37.19 \%$ & $26.46 \%$ & $33.97 \%$ & $27.23 \%$ & $27.45 \%$ \\
mgc_matrix_mult_a & $9.56 \%$ & $7.31 \%$ & $11.95 \%$ & $6.85 \%$ & $7.03 \%$ \\
\hline mgc_matrix_mult_b & $9.60 \%$ & $8.14 \%$ & $19.25 \%$ & $7.08 \%$ & $7.15 \%$ \\
mgc_pci_bridge32_a & $13.11 \%$ & $24.10 \%$ & $20.51 \%$ & $9.35 \%$ & $9.57 \%$ \\
\hline mgc_pci_bridge32_b & $8.55 \%$ & $6.69 \%$ & $21.39 \%$ & $6.03 \%$ & $6.23 \%$ \\
\hline Total Average & $19.26 \%$ & $21.95 \%$ & $25.37 \%$ & $14.04 \%$ & $14.24 \%$ \\
\hline
\end{tabular}

Table 2: Error metrics in all gcells and outlier gcells of different approaches. The table also shows percentage of local nets out of all the nets, and percentage of outlier local nets out of all the local nets.

\begin{tabular}{c|c|ccc|c|ccc}
\hline & & \multicolumn{3}{|c|}{ Error in all gcells } & & \multicolumn{2}{c}{ Error in outlier nets } \\
\hline & LN & PD & ST & ML & Outlier-LN & PD & ST & ML \\
\hline mgc_des_perf_1 & $31.77 \%$ & $97.48 \%$ & $80.19 \%$ & $25.83 \%$ & $0.85 \%$ & $84.38 \%$ & $33.84 \%$ & $15.77 \%$ \\
\hline mgc_des_perf_a & $20.82 \%$ & $50.59 \%$ & $18.01 \%$ & $7.40 \%$ & $0.91 \%$ & $78.22 \%$ & $36.21 \%$ & $26.41 \%$ \\
\hline mgc_des_perf_b & $22.64 \%$ & $85.65 \%$ & $37.14 \%$ & $14.24 \%$ & $0.32 \%$ & $87.43 \%$ & $33.64 \%$ & $25.66 \%$ \\
\hline mgc_edit_dist_a & $14.62 \%$ & $101.65 \%$ & $21.87 \%$ & $12.89 \%$ & $2.71 \%$ & $132.25 \%$ & $45.58 \%$ & $42.29 \%$ \\
\hline mgc_fft_1 & $32.26 \%$ & $95.12 \%$ & $75.93 \%$ & $27.58 \%$ & $0.88 \%$ & $44.89 \%$ & $33.02 \%$ & $16.10 \%$ \\
mgc_fft_2 & $29.06 \%$ & $92.25 \%$ & $50.17 \%$ & $24.47 \%$ & $1.50 \%$ & $46.69 \%$ & $30.47 \%$ & $19.45 \%$ \\
\hline mgc_fft_a & $15.68 \%$ & $79.40 \%$ & $12.83 \%$ & $7.63 \%$ & $0.56 \%$ & $73.90 \%$ & $61.31 \%$ & $61.21 \%$ \\
mgc_fft_b & $18.25 \%$ & $69.68 \%$ & $13.51 \%$ & $7.68 \%$ & $0.99 \%$ & $83.22 \%$ & $49.64 \%$ & $48.16 \%$ \\
\hline mgc_matrix_mult_1 & $31.92 \%$ & $105.72 \%$ & $83.89 \%$ & $27.45 \%$ & $1.04 \%$ & $41.50 \%$ & $34.26 \%$ & $15.48 \%$ \\
mgc_matrix_mult_a & $26.48 \%$ & $44.51 \%$ & $16.15 \%$ & $7.03 \%$ & $0.71 \%$ & $64.49 \%$ & $36.19 \%$ & $25.22 \%$ \\
\hline mgc_matrix_mult_b & $19.76 \%$ & $60.88 \%$ & $14.45 \%$ & $7.15 \%$ & $0.54 \%$ & $88.38 \%$ & $41.16 \%$ & $38.01 \%$ \\
mgc_pci_bridge32_a & $29.65 \%$ & $54.98 \%$ & $25.27 \%$ & $9.57 \%$ & $1.06 \%$ & $56.83 \%$ & $41.68 \%$ & $31.21 \%$ \\
\hline mgc_pci_bridge32_b & $25.12 \%$ & $59.78 \%$ & $12.93 \%$ & $6.23 \%$ & $0.37 \%$ & $57.39 \%$ & $39.36 \%$ & $40.18 \%$ \\
\hline Average & $24.46 \%$ & $76.74 \%$ & $35.56 \%$ & $14.24 \%$ & $0.96 \%$ & $72.27 \%$ & $39.72 \%$ & $31.17 \%$ \\
\hline
\end{tabular}

During testing, it outputs the mean prediction of each individual tree. Compared to decision tree, Random Forest is less likely to overfit its training data, and it is more robust to outliers and noise. (5) REPTree first builds a decision tree based on all the training instances and the feature vector, then it uses a reduced error pruning method to reduce the size of the tree by removing sections of the tree that provide little power to make prediction. Thus, the pruned tree generalizes better and gives higher accuracy because the complexity of the tree is reduced so it is less likely to overfit the training data.

We used the default settings in Weka in our experiments. In Table 1 we report the RAE over all gcells of these five different models. As can be seen from the table, RandomForest and REPTree have significantly lower error than the other three models.

RandomTree has the disadvantage that only a randomly picked subset of features are considered at each node, thus the model may not correctly utilize the most correlated feature(s). Even though each tree in RandomForest is very similar to a random tree, the ensemble mechanism ensures that the final prediction made by RandomForest is much better than an individual random tree. MultilayerPerceptron and DecisionTable perform worse than REPTree, possibly due to the fact that these two algorithms may overfit the training data. In contrast, REPTree reduces the chance of overfitting by pruning decision trees, thus increases the prediction accuracy.

While REPTree and RandomForest have similar error results, REPTree is faster to train. On average, RandomForest took $48 \mathrm{sec}-$ onds to train the model for a benchmark, while REPTree took 1.3 seconds. We identify REPTree as the suitable model for our problem and use it as our default algorithm hereafter.

\subsection{Comparison with Other Prior Work}

To put our machine learning approach into perspective, in Table 2 we compare the best Machine Learning (ML) algorithm identified above (REPTree) with the approaches proposed by other prior works.

Specifically, we compare with the Pin Density (PD) and Steiner Tree (ST) models explained in Section 2.3.

For linear regression in PD we used Weka [5] and as explained before, this generated the highest accuracy to implement this approach in our experimental setup. Furthermore, we used the same leave-oneout cross validation technique for all $P D$, and ML models during our training and testing. Leave-one-out cross validation has not been used for the ST model since the ST model does not require training.

In Table 2 Column 2 we report the percentage of Local Nets (LN) of all nets in a benchmark which is on average close to $25 \%$ of all the nets. First, we consider the RAE metric reported in Columns 3 to 5 . As can be seen, the ML model is significantly and consistently better than PD and ST across all the benchmarks. Even though the ST model is significantly better than the PD model, it is still worse than the ML model. The superiority of the ML may be caused by the fact that it can learn more knowledge about local route utilization from new features such as local pin density and radius-1 neighbors, as well as the interaction between all the features. Also, our estimated wirelength $(E W)$ feature works well as an estimate to Steiner wirelength because we observed that often times a local net inside a g-cell has a very small number of pins. 
Table 3: Runtime Comparison

\begin{tabular}{c|ccc}
\hline & \multicolumn{3}{|c}{ Runtime (minutes) } \\
\hline & PD & ST & ML \\
\hline mgc_des_perf_1 & 0.92 & 36.50 & 1.04 \\
mgc_des_perf_a & 0.85 & 22.87 & 1.18 \\
mgc_des_perf_b & 0.87 & 25.76 & 1.03 \\
mgc_edit_dist_a & 0.98 & 19.55 & 1.23 \\
mgc_fft_1 & 0.29 & 10.82 & 0.34 \\
mgc_fft_2 & 0.29 & 9.77 & 0.35 \\
mgc_fft_a & 0.27 & 4.99 & 0.51 \\
mgc_fft_b & 0.28 & 5.93 & 0.51 \\
mgc_matrix_mult_1 & 1.32 & 50.72 & 1.48 \\
mgc_matrix_mult_a & 1.29 & 40.93 & 2.12 \\
mgc_matrix_mult_b & 1.22 & 30.29 & 2.01 \\
mgc_pci_bridge32_a & 0.26 & 8.86 & 0.34 \\
mgc_pci_bridge32_b & 0.27 & 7.48 & 0.50 \\
\hline Average & 0.70 & 21.11 & 0.97 \\
\hline
\end{tabular}

\subsection{Runtime Comparison}

We observed that predicting local route utilization using the ML and PD models were much faster than prediction using the ST model. (Note, our final error measurements followed the same procedure for all methods and took approximately 2 minutes to complete for each benchmark regardless of the used model.) Therefore for runtime comparison, we only compare the time it takes to collect the data for each method and train and evaluate the models. On average, the data collection took $0.69,0.52$, and 0.95 minutes for the $\mathrm{PD}, \mathrm{ST}$, and ML models respectively. Therefore, the main difference in runtime resulted from evaluating the Steiner tree wirelength using FLUTE (in ST) versus training and testing the ML and PD models using Weka. Training and testing the PD and ML models were very fast, at most taking a few seconds to complete while Steiner tree computation using FLUTE was comparatively slow, taking at maximum 49 minutes to complete. As can be seen in Table 3 , the ST model took on average 21.11 minutes to complete, while both the PD and ML models took an average of less than 1 minute to complete. Our ML model achieved better results than the ST model in a fraction of the time.

\subsection{Comparison Among Outlier Local Nets}

In the final part of this experiment we compute a relative absolute error for a small subset of local nets which we define as "outlier" nets. We define a local net to be outlier if it is routed in more than one gcell. A gcell is then defined as outlier if it includes at least one outlier local net. Outlier local nets and gcells are first identified per design from the detailed routing data and then used to perform this experiment. In Table 2 we report the percentage of outlier local nets compared to all local nets in Column 6 which is a very small value (hence the name outlier). Here, we first compute a relative absolute error for each outlier local net (denoted by outnet) by computing and averaging the errors across its corresponding outlier gcells (denoted by outgcell). After computing an error per outlier local net, we then report the average of these errors across all outlier local nets. This is described in the formula below.

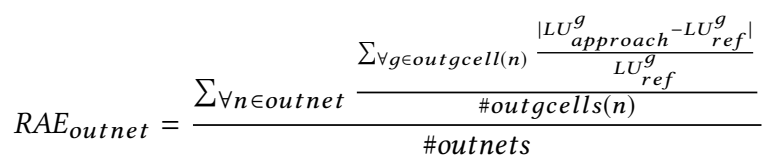

We note, this error is ultimately a net-based metric (to capture error across outlier local nets) while the previous error was a gcell-based metric (computed for each gcell).
The results for the error of outlier nets is reported in Columns 7 to 9 in Table 2. Here ML has consistently lower error than ST and PD. In one case for the $m g c \_$edit_dist_a benchmark and the $\mathrm{PD}$ approach, the error is higher than $100 \%$. This is because the predicted LU by the PD approach was significantly higher than the reference one per gcell.

Overall, we conclude that our ML model consistently generates the most accurate prediction compared with the other methods proposed by prior works.

\section{CONCLUSIONS}

We modeled the route utilization by local nets inside individual gcells using various machine learning algorithms. Compared to prior work, first the machine learning model was validated using detailed routing data of local nets and a commercial tool. Also it included new features such as steiner tree wirelength estimation, local pin density, neighborhood information, as well as interaction among the features. When ranking different features, we concluded that post-global routing features and radius-2 neighborhood features did not cause much difference and consistent improvement in modeling. We compared different machine learning algorithms and identified the most suitable one, and showed significantly lower modeling error compared to prior work.

\section{ACKNOWLEDGMENTS}

This research is supported by National Science Foundation under award 1608040 .

\section{REFERENCES}

[1] Olympus-SoC: Place and route for advanced node designs.

[2] I. S. Bustany, D. G. Chinnery, J. R. Shinnerl, and V. Yutsis. ISPD 2015 benchmarks with fence regions and routing blockages for detailed-routing-driven placement. In International Symposium on Physical Design, pages 157-164, 2015.

[3] W. J. Chan, P. Ho, A. B. Kahng, and P. Saxena. Routability optimization for industrial designs at sub-14nm process nodes using machine learning. In $A C M$ International Symposium on Physical Design, pages 15-21, 2017.

[4] C. Chu and Y. C. Wong. FLUTE: Fast lookup table based rectilinear steiner minimal tree algorithm for VLSI design. IEEE Transactions on Computer-Aided Design of Integrated Circuits and Systems, 27(1):70-83, 2008.

[5] E. Frank, M. A. Hall, and I. H. Witten. The WEKA Workbench. Online Appendix for "Data Mining: Practical Machine Learning Tools and Techniques". Morgan Kaufmann, Fourth Edition, 2016.

[6] C. Huang, C. Chiou, K. Tseng, and Y. Chang. Detailed-routing-driven analytical standard-cell placement. In Asia and South Pacific Design Automation Conference, pages 378-383, 2015.

[7] R. Kohavi. The power of decision tables. Machine learning: ECML-95, pages 174-189, 1995.

[8] T. M. Mitchell. Machine Learning. The Mc-Graw-Hill Companies, Inc, 1997.

[9] D. Shi and A. Davoodi. Improving detailed routability and pin access with 3D monolithic standard cells. In ACM International Symposium on Physical Design, pages 107-112, 2017.

[10] D. Shi, E. Tashjian, and A. Davoodi. Dynamic planning of local congestion from varying-size vias for global routing layer assignment. IEEE Transactions on Computer-Aided Design of Integrated Circuits and Systems, 36(8):1301-1312, 2017.

[11] H. Shojaei, A. Davoodi, and J. T. Linderoth. Planning for local net congestion in global routing. In ACM International Symposium on Physical Design, pages 85-92, 2013.

[12] Y. Wei, C. C. N. Sze, N. Viswanathan, Z. Li, C. J. Alpert, L. N. Reddy, A. D. Huber, G. E. Téllez, D. Keller, and S. S. Sapatnekar. GLARE: global and local wiring aware routability evaluation. In IEEE/ACM Design Automation Conference, pages 768-773, 2012.

[13] M. Wong, W. Liu, and T. Wang. Negotiation-based track assignment considering local nets. In IEEE Asia South Pacific Design Automation Conference, pages 378383, 2016.

[14] V. Yutsis, I. Bustany, D. G. Chinnery, J. R. Shinnerl, and W. Liu. ISPD 2014 benchmarks with sub-45nm technology rules for detailed-routing-driven placement. In ACM International Symposium on Physical Design, pages 161-168, 2014. 